\title{
Adaptive Shock Control Bumps
}

\author{
E. Jinks, P. J. K. Bruce ${ }^{\dagger}$ and M. Santer ${ }^{\ddagger}$ \\ Department of Aeronautics, Imperial College London, London, SW7 2AZ
}

\begin{abstract}
This paper describes a numerical investigation into the optimal design of adaptive shock control bumps (SCB) for transonic wings. A multi-disciplinary approach to optimization is utilized, combining structural and aerodynamic analysis to ensure that optimal adaptive SCB do not exceed material constraints whilst maintaining aerodynamic qualities. It is found that adaptive SCB can perform to within $95 \%$ of the non-structurally constrained bumps but over a much wider flight envelope. Two dimensional single crest bumps have been shown to match the performance of table top bumps originating from three dimensional SCB. Total pressure recovery has been successfully used as a performance metric and will provide valuable comparisons to wind tunnel experiments with a prototype adaptive SCB.
\end{abstract}

\section{Introduction}

$\mathrm{L}^{\mathrm{LOW}}$ control techniques have been an established part of aeronautics for many decades and have ranged from early stage wing fences to highly adaptable morphing wings. Shock control bumps (SCB), are one such type of passive flow control device which have shown promise for use on conventional supercritical wings and are particularly well-suited to natural laminar flow (NLF), wings. The large extent of favorable pressure gradient extending over the upper surface results in a large pocket of supersonic flow which is terminated by a strong near-normal shock wave at $\mathrm{x} / \mathrm{c} \approx 0.65$. Compared with earlier transonic airfoil designs the shockwave is further back to maximize the region of favourable pressure gradient. This increases the maximum Mach number on the upper surface to $M \approx 1.4$, increasing the strength of the shock induced adverse pressure gradient. This can negatively affect the health of the boundary layer and at worst, cause the flow to separate. The total pressure losses from a Mach 1.4 shock are significant and contribute to the wave-drag associated with transonic flight and form one of the bounds of cruise Mach number.

The SCB was a concept originally proposed in 19921 and in its original guise featured a flexible surface that could deform to create a bump beneath a shock. Throughout the EUROSHOCK II study, the adaptive bump idea was placed on hold whilst the aerodynamic characteristics of SCBs were explored. As an entirely structural project there have been results presented with a new family of bumps based upon possible shapes of an adaptive plate with particular focus to spring loaded actuators. However, an experimental aerodynamic analysis of these bumps was not carried out.2 The new SCB shape family aim was to minimize the energy term in the small deflection, Euler-Bernoulli linear theory. Other objective functions included maximum force criterion although there was only a slight difference in the force distribution. The geometries produced via the actuator optimization technique did show significant drag reductions compared to the clean ADIF NLF (natural laminar flow) airfoil (14\%) and similar reductions to the polynomial bump (15\%). Structural analysis of adaptive bumps has taken various forms, from optimization with respect to actuators ${ }^{2}$ to tube

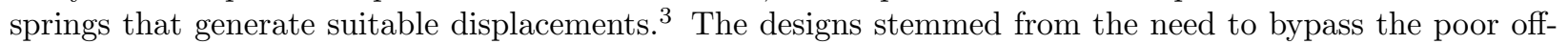
design characteristics of NLF airfoils. Significant levels of work have been conducted ranging from the early airfoil scale experimental $\sqrt{4}$ and numerical, $\frac{5}{5}$ studies to the most recent investigating of small-scale vortical patterns developed from $3 \mathrm{D}$ bumps 67

The shock structure shown in figure 1 is known as a bifurcated shock or $\lambda$-shock. The benefits of this type of shock are that the two stage compression is more efficient in reducing the Mach number in terms of total

* PhD Candidate, AIAA Member

${ }^{\dagger}$ Lecturer, AIAA Member

${ }^{\ddagger}$ Senior Lecturer, AIAA Member 


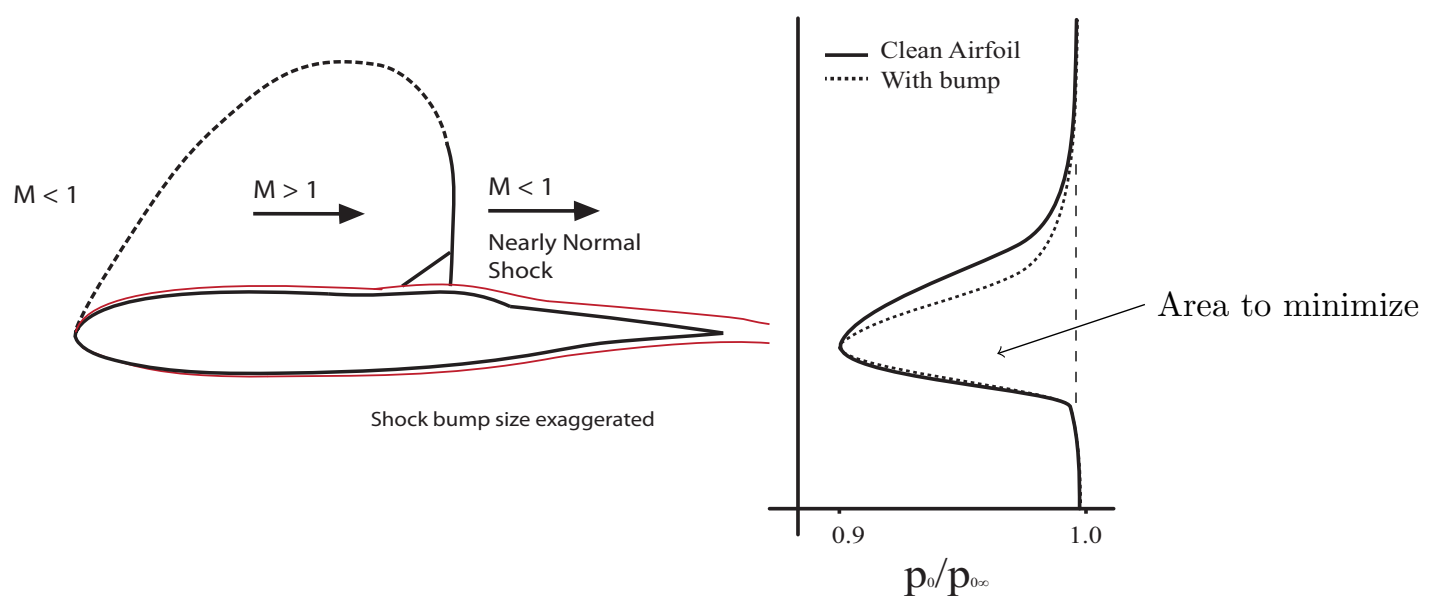

Figure 1. Typical shock structure and downstream total pressure recovery for transonic airfoil fitted with SCB. Shock bump enlarged for clarity.

pressure recovery. A close up of the structure is presented in figure 2 the increase in efficiency occurs because the strength of the normal shock at the rear leg is reduced. Typical bump heights are $0.4 \% c \leqslant h_{b} \leqslant 0.8 \% c$ with lengths of roughly $l_{b}=0.2 c$. To maximize this pressure recovery and hence decrease overall drag, the height of the triple point needs to be as high as possible. Experiments ${ }^{8}$ have shown heights of up to $0.06 \mathrm{c}$ are achievable in wind tunnel tests. $\frac{9}{9}$ This is limited by the maximum turning angle of the upstream supersonic flow and feasible bump lengths.

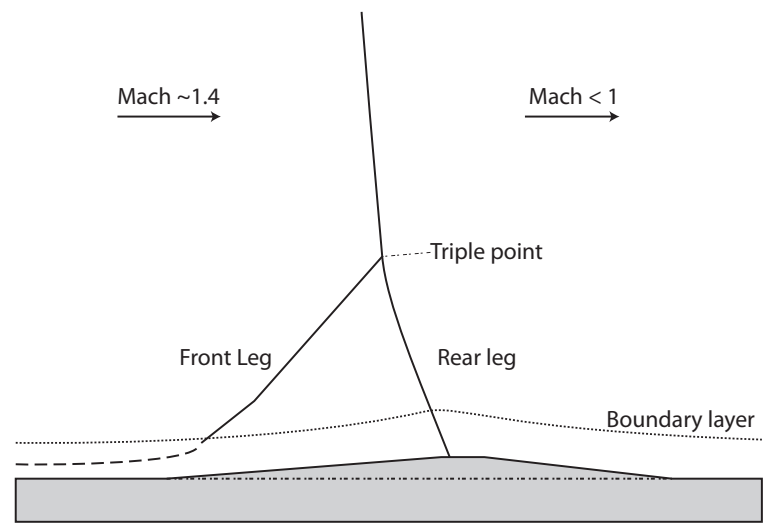

Figure 2. Typical $\lambda$-shock structure across an SCB

Much of the work regarding SCB has focussed upon static bumps and optimal design to ensure robust performance over a wide range of operating conditions; particularly $C_{l}$ and $M_{\infty}$. An SCB with suitable off-design performance is yet to be found without a corresponding sacrifice to on-design flow conditions. The adaptive SCB has the potential to negate the off-design performance drops associated with static bumps through the use of a morphing surface. Combining an adaptable SCB with an optimal design study, a physical wind tunnel model has been made for use in the supersonic facility at Imperial College London.

In this paper we aim to:

- Assess performance of bump shapes optimized for the mechanical limits of the material as well as the actuation system and aerodynamic performance;

- Discuss the relevant geometries for adaptable bump shapes.

These are carried out by means of a developed optimization framework that includes both aerodynamic and structural constraints. 


\section{Adaptive SCB Design}

The general shape and positioning of SCB have always been considered important variables and the key bump scale parameters are initial ramp angle and crest height, $\frac{10]}{10}$ these determine the structure of the $\lambda$-shock and hence the potential total pressure recovery.

There have been many optimization studies based upon buffet alleviation, L/D ratio and total pressure recovery. As the technology becomes more practically feasible it is timely to consider structural behavior. Incorporating structural optimization in tandem with aerodynamic analysis aids the feasibility of the technology. Designs have been developed which permit structural morphing, $2[11$ but the transient effects of bump deployment have not yet been considered.

\section{A. Optimization}

Optimization of the design required a multi-disciplinary approach combining a commercial finite element package (ABAQUS CAE ${ }^{12}$ ), and an open source CFD package (OPENFOAM ${ }^{13}$ ), around a central controller which utilized the gradient based fmincon function from the optimization toolbox in MATLAB ${ }^{14}$ The process is outlined in figure 3 and defined in equations 113. In the second iteration the air pressures are incorporated into the loading on the FE model.

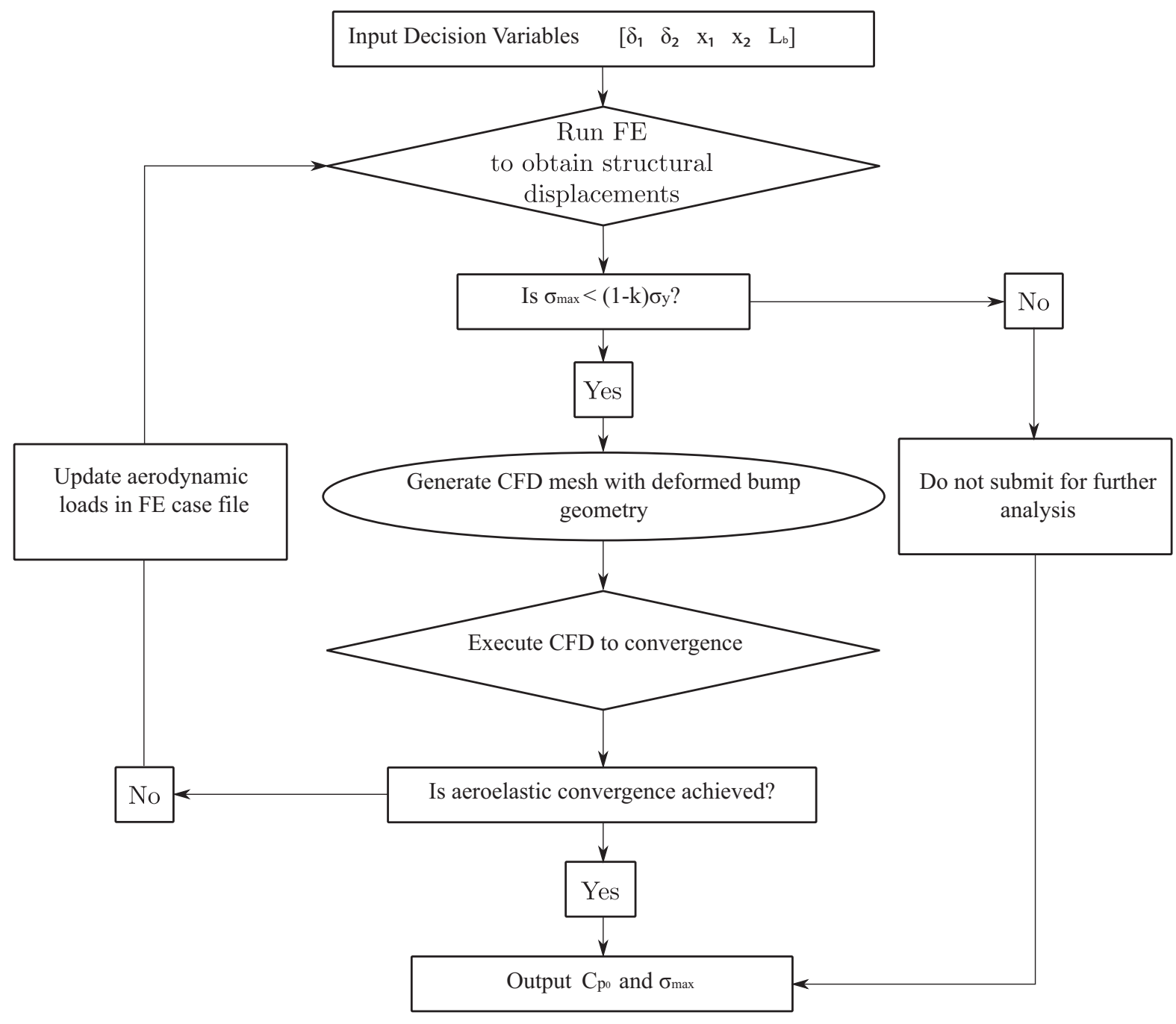

Figure 3. Developed aeroelastic optimization process for SCB. 


$$
\begin{gathered}
\left.\Delta C_{p_{0}}=\frac{1}{y_{d}} P_{0_{\infty}} \int_{0}^{y_{d}} P\left(1+\frac{\gamma-1}{2} \mathrm{M}^{2}\right)^{\frac{\gamma}{\gamma-1}}\right) \mathrm{d} y \\
\min \left\{\Delta C_{p_{0}}\right\} \\
\text { s.t. } \\
\sigma_{\max } \leq(1-k) \sigma_{y}
\end{gathered}
$$

In which $\Delta C_{p_{0}}$ is total pressure coefficient change between the free stream and a location downstream of airfoil. $y_{d}$ the height of the CFD domain, $\sigma_{\max }$ is maximum stress in FE analysis and $\sigma_{y}$ is the yield stress of the material.

The aim of the optimization is to minimize the loss of total pressure caused by the shockwave and compute optimum values for the decision variables listed in equation 4 and portrayed in Figure 4 . Total pressure recovery is chosen as the design metric as it allowed for greater distinction between viscous and wave drag effects than lift or drag based measurements. Total pressure measurements are also comparable to those taken from the wind tunnel described in Section IV] As adaptive SCB will be deployed in the cruise regime of a transonic aircraft, there will always be a significant total pressure loss across the range of Mach numbers of cruise.

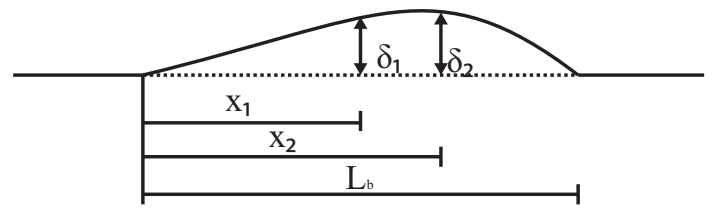

Figure 4. SCB decision variables used in the aeroelastic optimization.

$$
D=\left[\begin{array}{lllll}
\delta_{1} & \delta_{2} & x_{1} & x_{2} & X_{1}
\end{array}\right]
$$

A 2D bump shape was chosen because of the superior on-design performance when compared to 3D bumps $\frac{15}{15}$ Off-design, the capabilities of $2 \mathrm{D}$ geometries deteriorate significantly when the shockwave moves fore and aft with unsteady freestream conditions. The extensive justifications throughout the EUROSHOCK II project $\frac{16}{16}$ also allude to the greater drag reduction potential of two dimensional bumps with three dimensional bumps offering greater flow control characteristics. If the shape of the bump is to be adapted throughout flight with changing lift requirements during cruise then the potential drag savings are greater with a $2 \mathrm{D}$ bump as well as the reduction in computational cost.

The SCB were limited to shapes in within the loaded beam family: this ensures a shape that is physically possible within material and airframe integration constraints as well as meeting the geometrical requirements of flow-structure interaction. Figure 5107 nicely summarizes the number of bump families available with a minimal number of design variables, height and crest location are key with other variables reported to have minor effects. ${ }^{9}$ The length of the bump was chosen to be $l_{b}=0.2 c$ based upon typical values found previously $10\left[16\right.$ For a chord length of one metre this meant $l_{b}=200 \mathrm{~mm}$ which is a suitable size for wind tunnel testing, Section IV] The system has been artificially limited to two actuation points as the majority of $2 \mathrm{D}$ bump shapes could be achieved without the complexity of more. The three stages created by two loading points incorporate the initial ramp and two sections for the gradual turning of flow to return it to a stream-wise direction. An extensive experimental study ${ }^{\sqrt{9}}$ suggested that this table-top design is among the most promising for drag reduction alone so will be used as a starting point for the optimization prior to more specific designs.

\section{B. Structural Analysis}

Including a finite element analysis provides the structural constraint and ensures continuity if rotation between the airfoil and the modified bump section. An optimization study $\frac{18}{18}$ found the family of loaded beam geometries offers a high enough wave drag reduction with minimal viscous drag increase when compared to wedge type bumps, concave polynomials and high order polynomials. The shape continuity associated with 


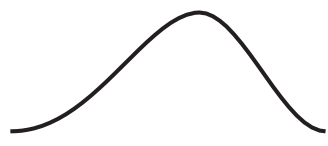

Loaded Beam

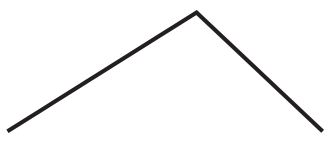

Triangular Shape

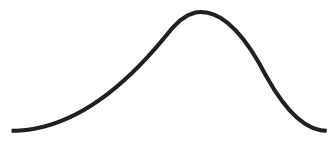

Polynomial

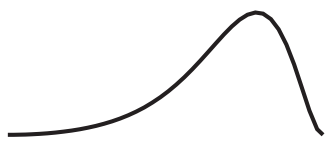

Concave Polynomial

Figure 5. Minimal design variable bump shapes 17

beam bending shapes led to less severe changes in pressure distribution along the top surface which will not only lend itself to material constraints but also handling constraints of the aircraft when adaptive bumps are deployed.

A geometrically nonlinear, quasi-static finite element analysis was setup to model the plate section with built-in ends and constant loads across the span. A mesh convergence study was carried out and the results presented in figure 6. The results show a significant number of elements are required in order to reach a satisfactory percentage of the analytical solution. The output consisted of deflection and von Mises equivalent stress for each shell element. The necessary repetition of deployment is essential and so the system must remain in the elastic regime to avoid plastic deformation of the material. The yield stress of the material represents the upper limit and is given at $525 \mathrm{MPa}$ with a $10 \%$ safety margin corresponding to the material properties of AL-7075-T6. $(\mathrm{E}=72 \mathrm{GPa}, \nu=0.33, \mathrm{t}=0.6 \mathrm{~mm})$. This was applied to ensure that any imperfections within the material were accounted for as well as to help reduce the effects of cyclic loading when in potential airframe use. Bump shapes that met this criteria were then used to create the mesh used for CFD.

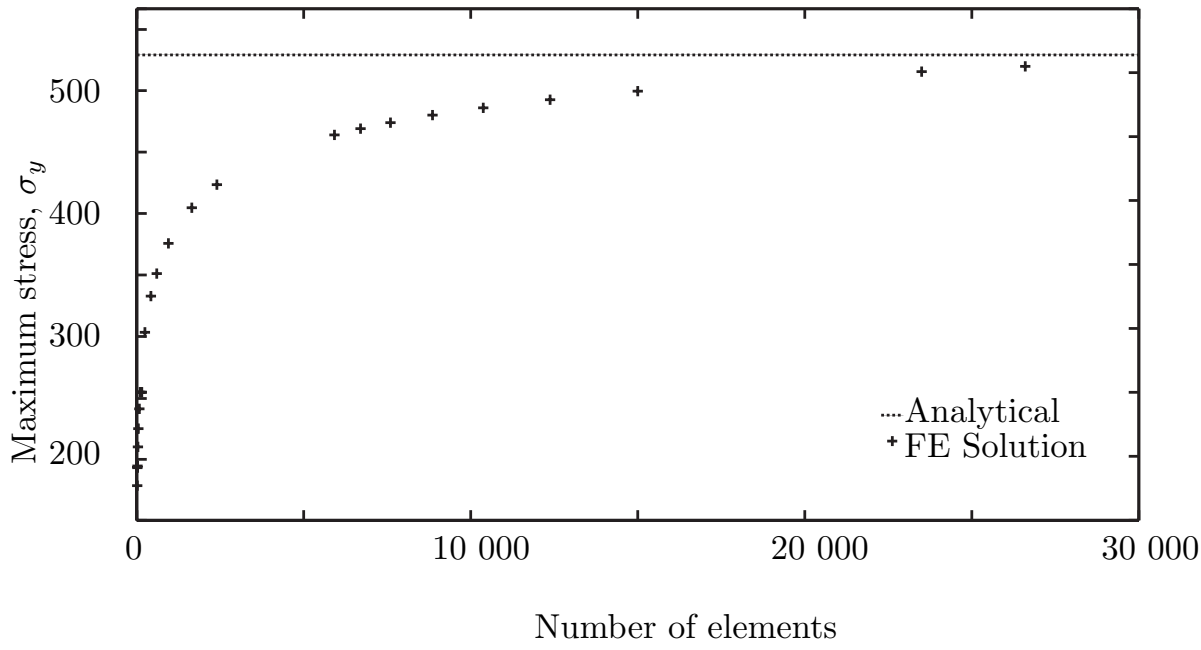

Figure 6. Finite Element mesh convergence

\section{Aerodynamic Analysis}

The CFD analysis was completed using the sonicFoam ${ }^{13}$ solver within OpenFoam that is designed for applications to transonic flow. It solves the Reynolds Averaged Navier-Stokes equations through the finite volume method. The turbulence model used is k- $\omega$-SST, combining the near wall properties of K- $\omega$ and the freestream handling of $\mathrm{K}-\epsilon$. The model also lends itself to the adverse pressure gradients apparent around the shock region. The RAE2822 supercritical airfoil is used to provide a proven testbed for the aerodynamic aspect of the optimization. The curvature over the top surface of the airfoil is not particularly severe with a 
maximum thickness of $0.121 \mathrm{c}$ occurring at $x=0.5 \mathrm{c}$ which lends itself to a larger variety of shock locations for subtle changes in pressure distribution than thicker airfoils.

Mesh refinements were added to the boundary layer and expected shock region to better capture the flow as shown in figure 7, in order to increase solution accuracy. Wall functions were avoided due to the high likelihood of bump induced separation which the k- $\omega$-SST model should capture. In order to ensure independence of the results from the mesh, numerous algorithms were employed to design a mesh tailored to airfoils. The relatively small changes of airfoil geometry, typically $0.02 \%$ c between bump iterations, meant that mesh optimization could be completed outside of the aero-structural optimization resulting in a reduction of the computational time. Mesh independence tests were carried out adjusting the cell density, airfoil surface coverage and overall domain size to ensure that the boundaries were sufficiently far away to not interfere with the solution. A structured C-grid setup with quad-elements was chosen with upper and lower boundaries placed 7 chord lengths away with an outlet boundary condition placed 10 chords lengths downstream which allowed sufficient room for the wake to grow and pressure waves to propagate freely.

The mesh was first generated using a linear transfinite interpolation scheme to produce an initial grid based upon the boundary grid node distribution at the surface of the airfoil 11 This basic mesh was then elliptically refined based upon the solution of a set of partial differential equations which were known to produce a grid with smoothly varying cell sizes and slopes of grid lines $\frac{19}{19}$ The orthogonality of the grid lines at the airfoil can be controlled within the elliptic refinement alongside the implementation of Neumann orthogonality boundary conditions. This was done to calculate the position of the boundary node along the airfoil surface to ensure orthogonality. The mesh produced from this series of algorithms successfully passed OPENFOAM's built in mesh checking function, checkMesh.

\begin{tabular}{c|c|c|c} 
Mesh generation method & Max non-Orthogonality & Mean non-Orthogonality & Max Skewness \\
\hline Linear TFI & 70.55 & 18.33 & 1.93 \\
TFI + elliptical refinement & 70.57 & 13.40 & 2.70 \\
Neumann boundary conditions & 23.30 & 5.96 & 0.30
\end{tabular}

Table 1. Mesh refinement effects 11

The case was decomposed via the SCOTCH decomposition method which evenly distributed cells across the specified number of processors, this was deemed the most efficient method of decomposition within OPENFOAM. A converged solution of the clean RAE2822 airfoil was used as an initial starting condition for each of the bump analyses which were then mapped to the new mesh at each iteration. Each CFD simulation within the optimization was then run to convergence, the convergence criteria were set for when the residual for velocity fields were below $10^{-7}$ and pressure fields $10^{-18}$ for each time step. Post-processing of the solution required the computation of the total pressure field as this was not calculated or stored at each time step in order to speed up the computation times.

This mesh, appropriate boundary conditions and the solver were combined in order to complete a study of the clean wing setup. The results have been compared to a NASA study on the same airfoi ${ }^{20}$ with comparable shock position and surface pressure distribution. In order to make best use of the SCB the freestream Mach number was increased to $M_{\infty}=0.78$ which produced a strong shock $M_{\text {shock }}=1.4[4][17|21| 22$ This strength of shock wave was chosen as it represents a maximum upstream Mach number prior to downstream separation. Post-shock separation usually occurs with upstream Mach numbers around $M_{\text {sepn }} \approx 1.5$. The validation results in figure 7 show that the model is a suitable representation to the experiment based upon the location of the shockwave. The CFD placed the shock at $x_{s}=0.582 \mathrm{c}$ calculated by the interpolation between the pre and post shock pressures. The experiment ${ }^{20}$ showed a more gradual pressure rise downstream which has lengthened the interaction region; with the same technique, the position of the shock was $x_{s}=0.591 c$. This variation of less than $1 \% \mathrm{c}$ is deemed sufficient with the majority of the cases ${ }^{20}$ placing the shock within $2 \%$ c. The minimum pressure is within $7 \%$ of the experimental value which was measured to within $0.26 \%$ error. The validations in the previous studies also failed to capture this peak value however the remainder of the flow field was captured well.The region of low pressure at the nose was not captured well and so further work is being carried out to evaluate this however the main criteria of shock location and strength are within suitable margins of the experimental data. These are the important criteria that must be upheld. 


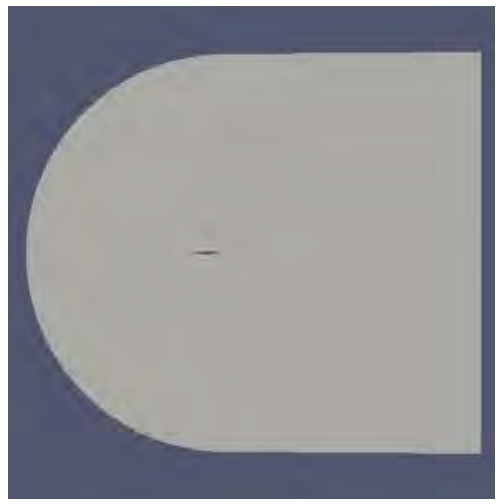

(a)

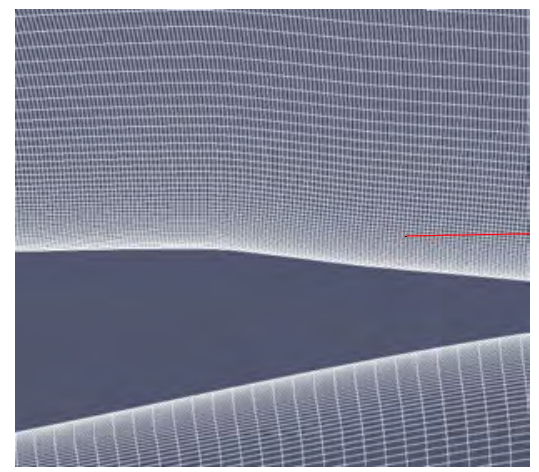

(b)

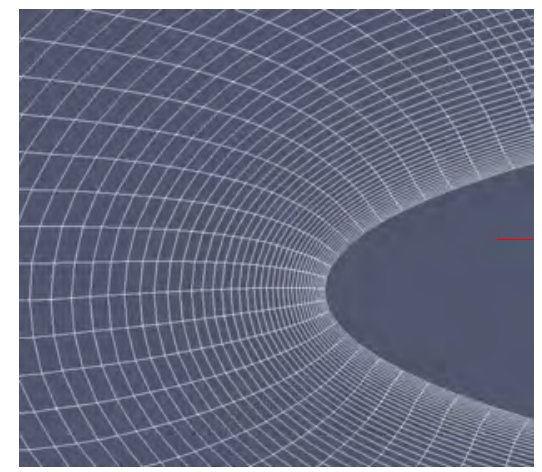

(c)

Figure 7. (a) Typical C-mesh for airfoil. (b) Refined mesh around shock location. (c) Refined mesh at nose of airfoil.

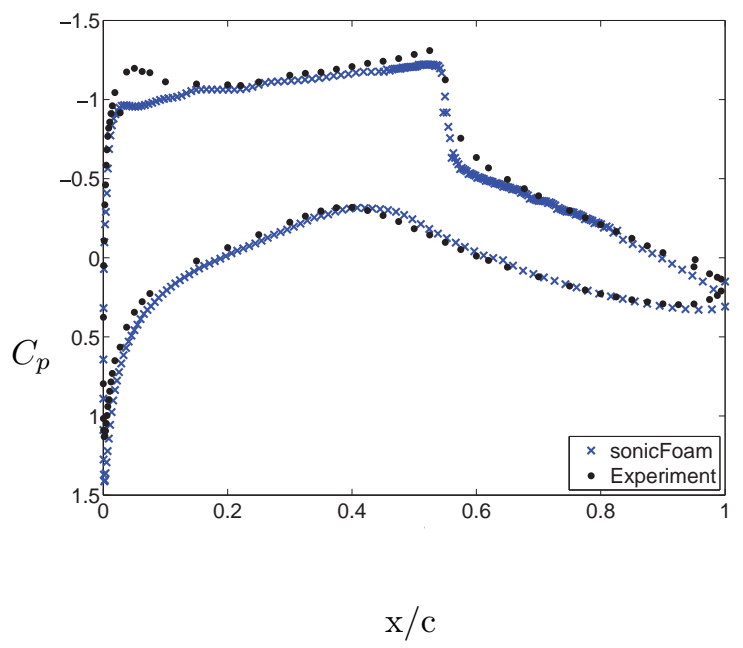

Figure 8. Pressure profile comparison of RAE2822 airfoil $M_{\infty}=0.73, \alpha=3.19^{\circ}$ 

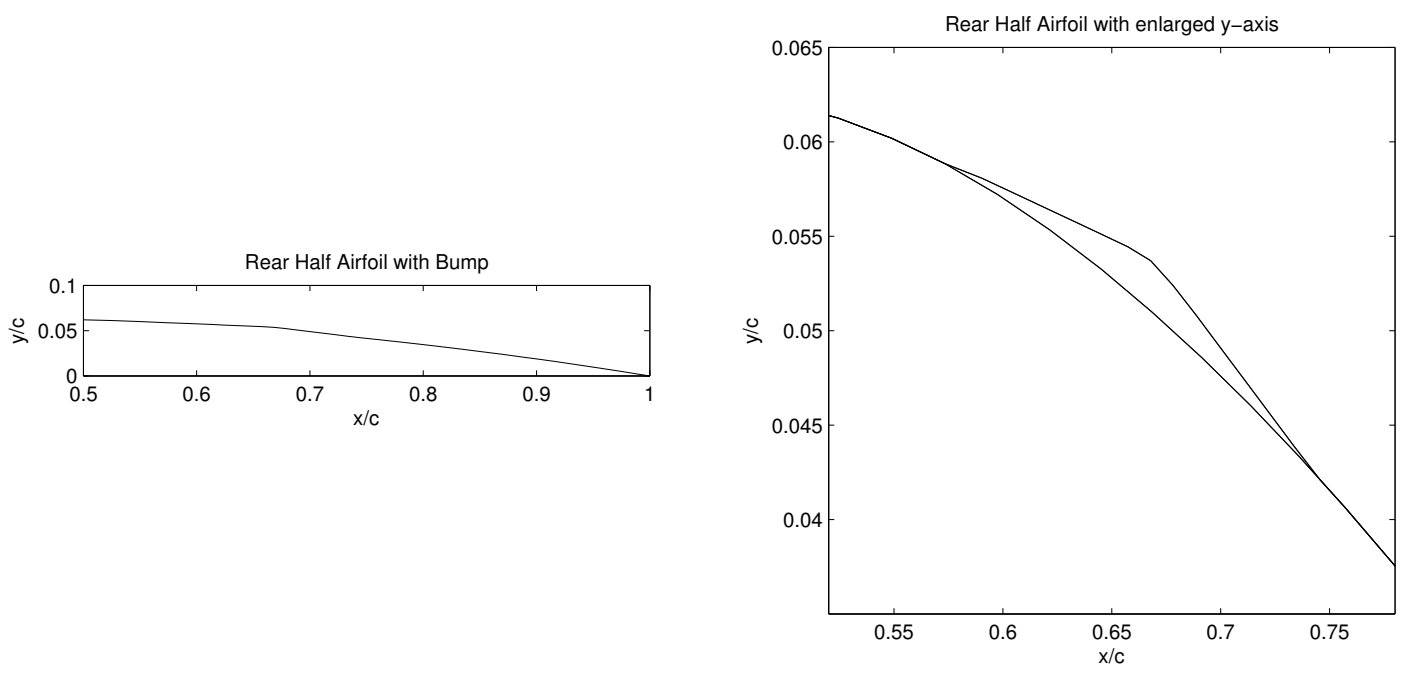

Figure 9. Rear half of airfoil with optimal bump, (table 3 w/ Constraint).

\section{Results for Static SCB}

With the greater investigation of three dimensional bumps shapes in recent years $\underline{6,23}[7]$ the simple SCB shapes shown in figure 5 have been expanded to include table tops and shoulder radii which are now recognized as important design variables through experimental laser diagnostic methods, PIV and LDA. The flow structure can be analysed around these complex three dimensional SCB with these methods and as such the fine tuning of these shapes can be investigated and validated. This has meant that it is now possible to evaluate much more of the flow field surrounding SCB and the purpose of bumps is moving away from only shock control devices but more general flow control.

The optimization stages now appear to be split into two scales, the first focussing on large-scale flow features such as bump height and crest location and smaller scaled features such as shoulder ramp angle. This approach, necessary with 3D bumps, was applied to the infinite span adaptive 2D bumps in order to ensure a suitable table top design in order to minimize the effects in the boundary layer whilst maintaining an efficient bump with respect to pressure. The health of the boundary layer after the bump is integral in order to recover the maximum amount of total pressure downstream of the airfoil. This will be discussed further in Section IIIC

In order to reduce computational time a pseudo-transient approach was employed which meant that the flow effects were not included in the structural analysis until a time-converged solution was found.

The original shock location for the free stream conditions $M_{\infty}=0.78 \quad \alpha=3.19$ was located at $x_{\text {shock }}=0.68 \mathrm{C}$. The results showed that the shock underwent an average movement of $1-2 \% \mathrm{C}$ for an arbitrarily placed bump suggesting their suitability for shock holding.

\section{Optimal Bump Geometry}

The bump geometries produced by the optimization process were found to be heavily dependent upon the initial conditions submitted to the process. The search criteria were then widened in order to explore the realm of the domain further however this still resulted in local minima very close to the initial conditions. The reason for such seemingly limiting behavior is that the structural constraint was limiting the height of the bumps to a level below that of aerodynamic optima shown previously!24 This opened a broad family of optimal bumps $^{2}$ that offer the maximum aerodynamic benefits with feasible material constraints. For the static bumps the study had two aims, the first was identifying the behavior of the optimization process due to the structural constraint and the second focussed upon the flow structure surrounding the bump. 


\section{A. Structural Constraint Limitations}

The optimization process in figure 3 was completed with and without the structural constraint in order to view the effect directly. Starting from an initial condition as described in Table 2 the optimizer was allowed to explore the design space in order to minimize the total pressure lost downstream. The values of each iteration are shown in figure 10 with the constrained problem converging to a local minima with a fewer number of iterations. The final, optimal bumps are presented in Table 3 and the difference is appreciable. The sketch in figure 11 shows the change of the optimal SCB geometry with and without the material restriction.

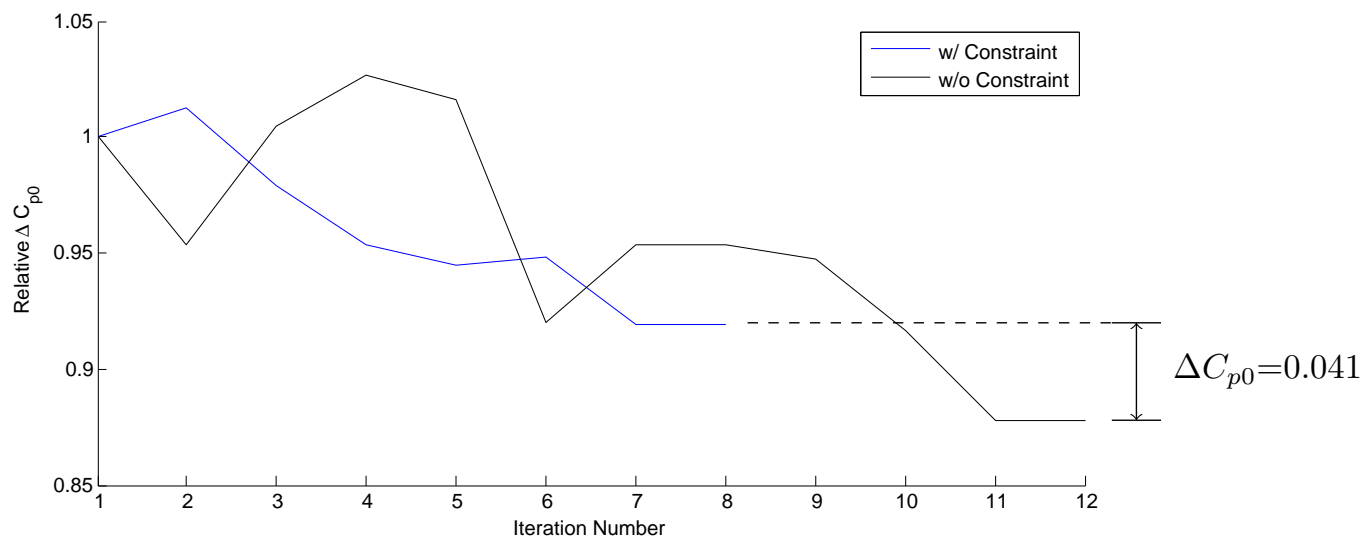

Figure 10. Total pressure loss, $\Delta C_{p 0}$, for optimizer iterations with and without the structural constraint. Iteration 1 represents the initial condition.

By comparing the crest height of these bumps it can be seen that they are both within the range of previously found values, $0.4 \% \mathrm{c}$ and $0.8 \% \mathrm{c} 225$ However these values did not take into account material limitations which have been shown in this study to restrict the crest height of the bumps.

\begin{tabular}{c|c|c|c|c|c|c}
$x_{1}\left(\% l_{b}\right)$ & $x_{2}\left(\% l_{b}\right)$ & $\delta_{1}(\mathrm{~mm})$ & $\delta_{2}(\mathrm{~mm})$ & $X_{1}$ & $\sigma_{\max }(\mathrm{MPa})$ & $\Delta C_{p 0}$ \\
\hline 0.55 & 0.622 & 4.5 & 4.5 & 0.59 & 373.69 & 1
\end{tabular}

Table 2. Initial conditions for structural constraint test. The cases presented in Tables 3 and 5 are normalized against these initial conditions

\begin{tabular}{c|c|c|c|c|c|c|c} 
& $x_{1}\left(\% l_{b}\right)$ & $x_{2}\left(\% l_{b}\right)$ & $\delta_{1}(\mathrm{~mm})$ & $\delta_{2}(\mathrm{~mm})$ & $X_{1}$ & $\sigma_{\max }(\mathrm{MPa})$ & $\Delta C_{p 0}$ \\
\hline w/ Constraint & 0.56 & 0.63 & 4.75 & 4.47 & 0.59 & 387.28 & 0.919 \\
w/o Constraint & 0.61 & 0.70 & 5.91 & 6.56 & 0.68 & $\gg \sigma_{y}$ & 0.878 \\
w/ Single Load & 0.56 & - & 4.75 & - & 0.59 & 372.2 & 0.938
\end{tabular}

Table 3. Optimal bump shapes for structural constraint test. Plastic deformation and material failure were not included in the FE model to speed up optimization.

During the iterations of the optimization process, the optimal bumps produced were seen to remain very close to the initial conditions. Manual tuning of the decision variables was undertaken to force the process to look further afield in order to explore the optimization domain. The optimal bump variables remained close to the initial conditions in all except the unconstrained case suggesting that the structural constraint is an important factor with respect to shaping the optimal bump geometry.

The movement of the unconstrained bump away from the initial condition highlights that a poorly designed and located unconstrained bump is worse than a poorly positioned constrained bump. Therefore the tolerances for a structurally optimized bump seem far greater than a unconstrained bump. This larger envelope for efficient design will be beneficial to the final applications on aircraft wings. 
Initial Condition

w Constraint

w/o Constraint

Figure 11. Sketch comparing bump shapes

The result produced from the unconstrained approach led to a bump with a larger front face for the shock to straddle and therefore a higher triple point and larger lambda. The effect of this can be seen in figure 12 which shows the incremental increase in total pressure recovery as bump height is increase, particularly between $0 \leq h_{d} \leq 0.05$ where the larger the bump, the higher the total pressure recovery downstream of the airfoil. The relative area changes are measured by the $\Delta C_{p 0}$ value in Table 3 and against the datum case in Table 4

From figure 12 the beneficial effects of SCB can be easily identified with the significant reduction in total pressure loss. The area that represents the total pressure loss has been reduced by $15 \%$ with the constrained bump and $19 \%$ without the structural constraint. However this was an expected result. If the flow were to deviate or $M_{\text {cruise }}$ change as often required, the shock position would alter and the higher bump would have a much larger penalty due to its presence within the boundary layer. Not only would the smaller, constrained bump have less of an effect due to the obstruction in the flow, the existence of many local minima nearby offers a very wide operating envelope for a given constrained bump design. As well as the lower bump alleviating some of the off-design deterioration it is adaptive and would be able to adjust to the new flow conditions and perform to within $5 \%$ of a static bump that was optimised for the new flow conditions.

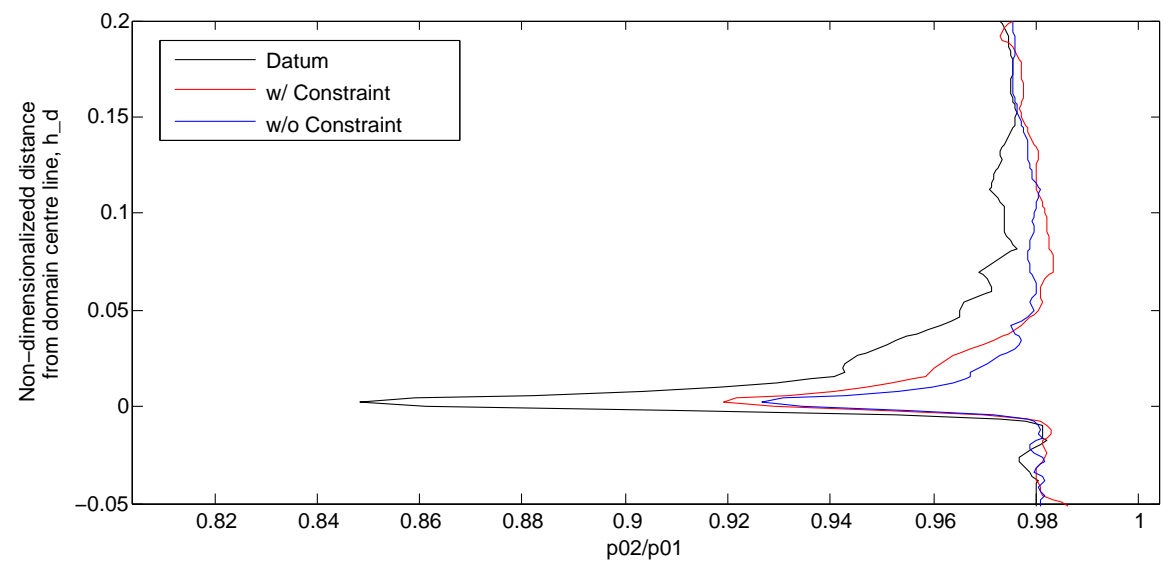

Figure 12. Downstream pressure profiles comparison between clean, constrained and unconstrained bump cases.

\begin{tabular}{c|c|c} 
Case & $\Delta C_{p 0}$ & Relative to Datum \\
\hline Datum & 0.0484 & 1 \\
w/ Constraint & 0.0414 & 0.85 \\
w/o Constraint & 0.0396 & 0.81
\end{tabular}

Table 4. Numerical values for $\Delta C_{p 0}$ with reference to $M_{\infty}=0.78, \alpha=3.19^{\circ}$ 


\section{B. Single Crest SCB}

The table top feature, which has been a significant feature of the most recent three dimensional bumps, has become less prominent during the two dimensional optimizations which have converged to a near single peak geometry. This suggests that not all design protocols are applicable to both sets of shock control bumps $(2 \mathrm{D} / 3 \mathrm{D})$ and could be a characteristic of their differing roles. Figure 13 highlights the subtle differences between a single load and the optimal design from Table 3 The differences in the diagram are slight however the crest of the optimal bump is located $5.4 \mathrm{~mm}$ further back and $0.03 \mathrm{~mm}$ higher due to the effect of the second loading point. This results in a $\Delta C_{p 0}=0.938$ which means that less total pressure was recovered than the optimal constrained bump (0.919). The optimization process for a single crest bump would be significantly less computationally expensive and would lead to a simpler actuation system for the experimental model.

The single crest concept supports the assertion that two dimensional bumps are control devices that should be used primarily to control the shock structure. This is in contrast to three dimensional bumps that are able to impart flow structures to the boundary layer much more readily, giving greater general flow control over the rear half of the airfoil.

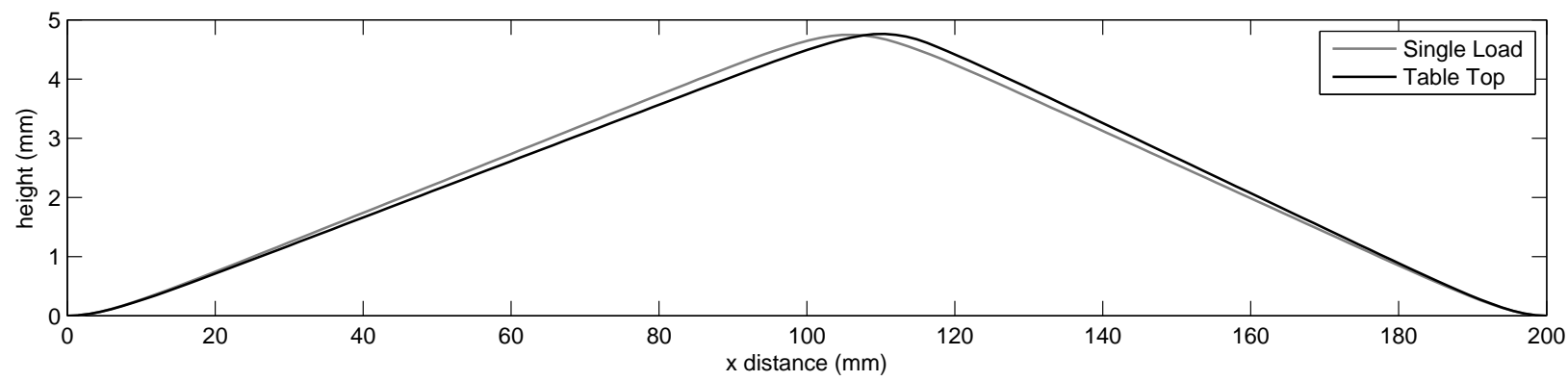

Figure 13. Comparison between single displacement and table top design bump geometries

\section{Boundary Layer Interaction}

The boundary layer is a significant feature within the $\lambda$-shock and was subject to significant change when a bump is deployed. From a constrained bump optimization analysis with initial conditions, Table 5 the boundary layer profiles have been plotted in figure 16 alongside the datum airfoil case for comparison. This local minimum has shown the potential of a lower bump a height similar to previous studies. ${ }^{25}$ Also of note is the initial table top condition having been removed in favour of a single crest SCB. The boundary layer profile locations were selected for their positions with respect to key bump features. From the most upstream location at $\mathrm{x}=0.5 \mathrm{c}$ the velocity profile appears to have a very similar shape although lower free stream velocity than the datum case. This could be due to the presence of the bump being known by upstream propagation of pressure waves through the subsonic section of the boundary layer. In order to confirm this idea high frequency pressure measurements at the wall are proposed as a measure to see if any similar fluctuation patterns are observed at the corresponding datum and bump shock conditions.

\begin{tabular}{c|c|c|c|c|c|c|c} 
& $x_{1}\left(\% l_{b}\right)$ & $x_{2}\left(\% l_{b}\right)$ & $\delta_{1}(\mathrm{~mm})$ & $\delta_{2}(\mathrm{~mm})$ & $X_{1}$ & $\sigma_{\max }(\mathrm{MPa})$ & $\Delta C_{p 0}$ \\
\hline Initial Condition & 0.5 & 0.25 & 4.0 & 4.0 & 0.55 & 309.26 & 0.917 \\
Local Minima & 0.51 & 0.651 & 4.39 & 3.44 & 0.54 & 303.96 & 0.793
\end{tabular}

Table 5. Structural constraint at bump start location, $X_{1}=0.55 c$, reference to initial condition bump, table 2

The profile extracted prior to the bump at $\mathrm{x}=0.55 \mathrm{c}$ sits at the starting position of the SCB, the smearing of the velocity away from the wall as the flow reacts to the initial change in slope and the corresponding pressure waves that reached the surface either side of the start point. At $\mathrm{x}=0.6 \mathrm{c}$ after the front leg of the $\lambda$-shock the flow is subject to continuously changing slope up to a maximum of $3^{\circ}$. The greater velocities at the top of the $\mathrm{x}=0.6 \mathrm{c}$ graph support the expected boundary layer compression as the flow adjusts to the 
new direction as enforced by the bump. The profiles begin to become less full which indicates the boundary layer energy moving further away from the wall. This was most likely a combination of boundary layer thickening due to the adverse pressure gradient across the shocks as well as the curvature of the geometry.

The curvature of the loaded beam family of geometries are much more sympathetic to boundary layers than the sudden deformation of a triangular shape. The effect at location $\mathrm{x}=0.55 \mathrm{c}$ would have been much more severe with a sudden angle change with a larger loss of momentum in the near wall region. The gradual increase in curvature smoothes the adverse pressure gradient across the oblique shock. The effect of the smeared front leg shock is also beneficial to the material as the loading is spread across the built-in ends of the bump which are the most highly stressed regions. As the flow is compressed due to the turning, the skin friction increases ${ }^{27}$ on the front surface of the bump. This could potentially increase the viscous losses caused by the bump which are visible in figure 12 in the region directly downstream of the airfoil.

The shockwave was located much further downstream on the bump than expected (figure 14) with the crest of the bump preceding the rear leg of the shockwave. This was not something that conformed to previous literature and could be attributed to the combination of an airfoil study with the goal of maximising total pressure recovery. The arc of the sonic line and the surrounding pressures that define where the global sonic line is positioned could be too far into the freestream for the near-wall SCB to affect.

The area of supersonic flow around the crest of the SCB expands the flow and accelerates it to form another $\lambda$-shock structure at the foot of the main rear leg. This secondary $\lambda$-shock rear leg remains perpendicular to the wall for a distance of $0.03 \%$ c which coincides with an increase in $p_{02} / p_{01}$ recovery in figure 12. This double $\lambda$ structure may be an effect of optimising the bump for maximum total pressure recovery. By holding the shock downstream of the crest it causes curvature of the shock as well as increasing the height of the triple point. This would further increase total pressure recovery by covering a larger area into the freestream. Careful balancing would be required between the level of expansion and resultant boundary layer thickening with the increase in pressure recovery. Enforcing another $\lambda$ structure post crest would reduce the adverse pressure gradient further as long as the mach number in this region did not exceed that of the main rear leg, $M_{\text {shock }} \leq 1.4$.

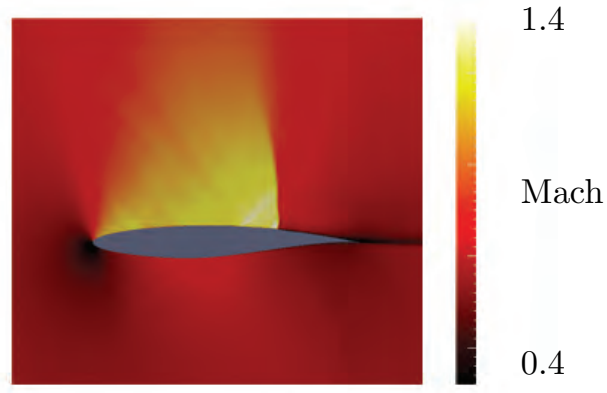

(a)

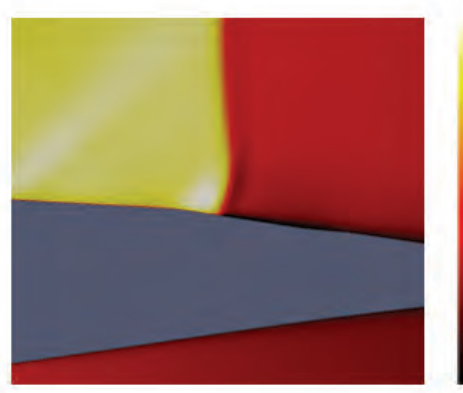

Mach

(b)

Figure 14. (a) Mach field view. (b) Zoom view of Bump region.

\section{Continuing Work: Experimental Adaptive Shock Control Bumps}

Some of the issues presented in previously are more reliably evaluated experimentally where the restrictions of computational models and resources are not an issue. Placing shock control bumps on the flat surface of the wind tunnel makes it easier to take good quality measurements rather than being restricted to the confined space within airfoils. The analysis of the near-bump flow can be investigated on a much smaller scale through the use of optical techniques and will aid the validation of optimal bump shapes.

Due to a combination of the findings in Section B and mechanical practicality a single crest beam shape was created and the result is shown in Figure 17. The finite element analysis captures the shape of the bump well however there are some slight differences at approximately $0.65 l_{b}$. This has been attributed to the edges 


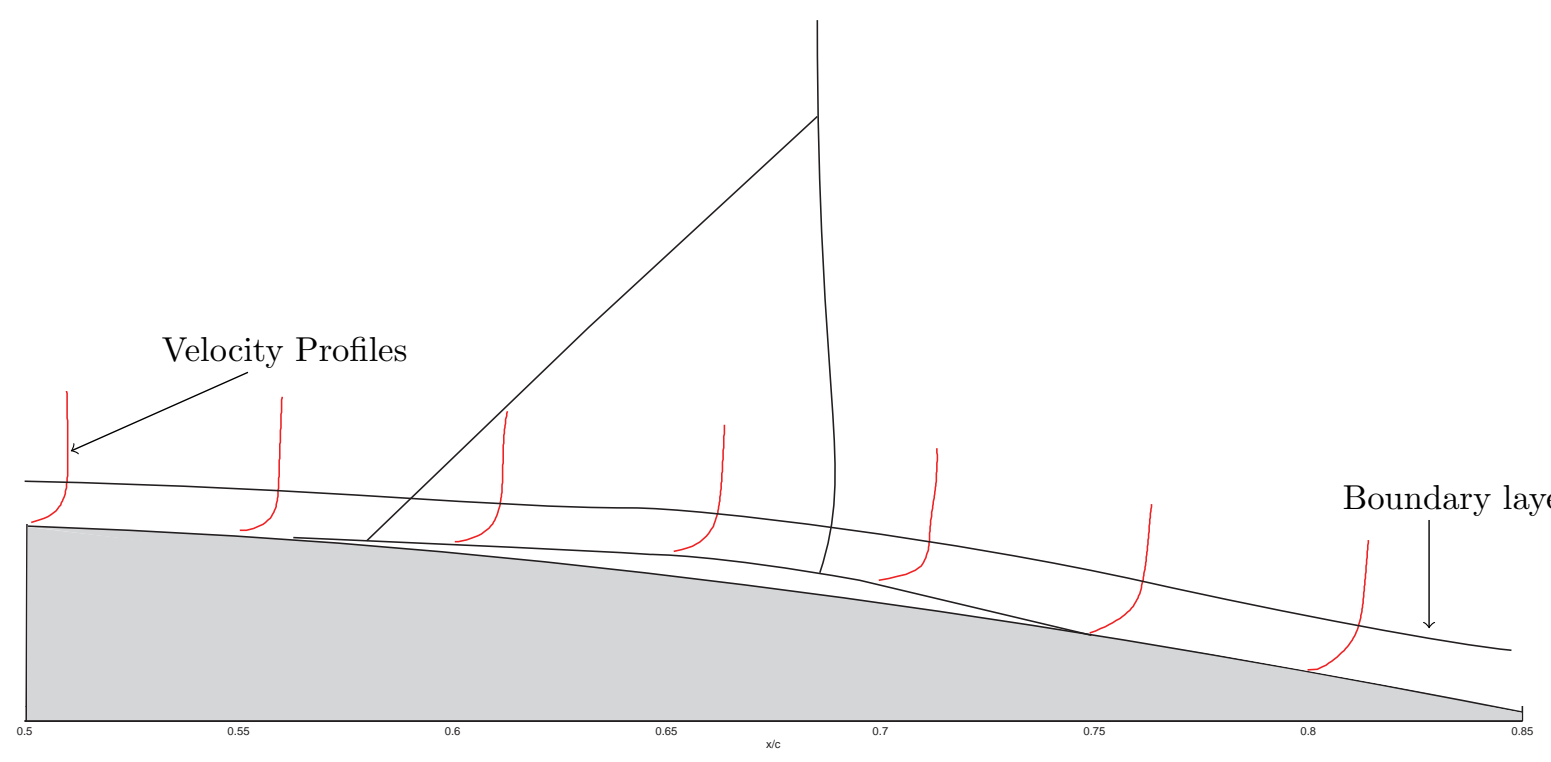

Figure 15. Boundary layer variation across a typical SCB.
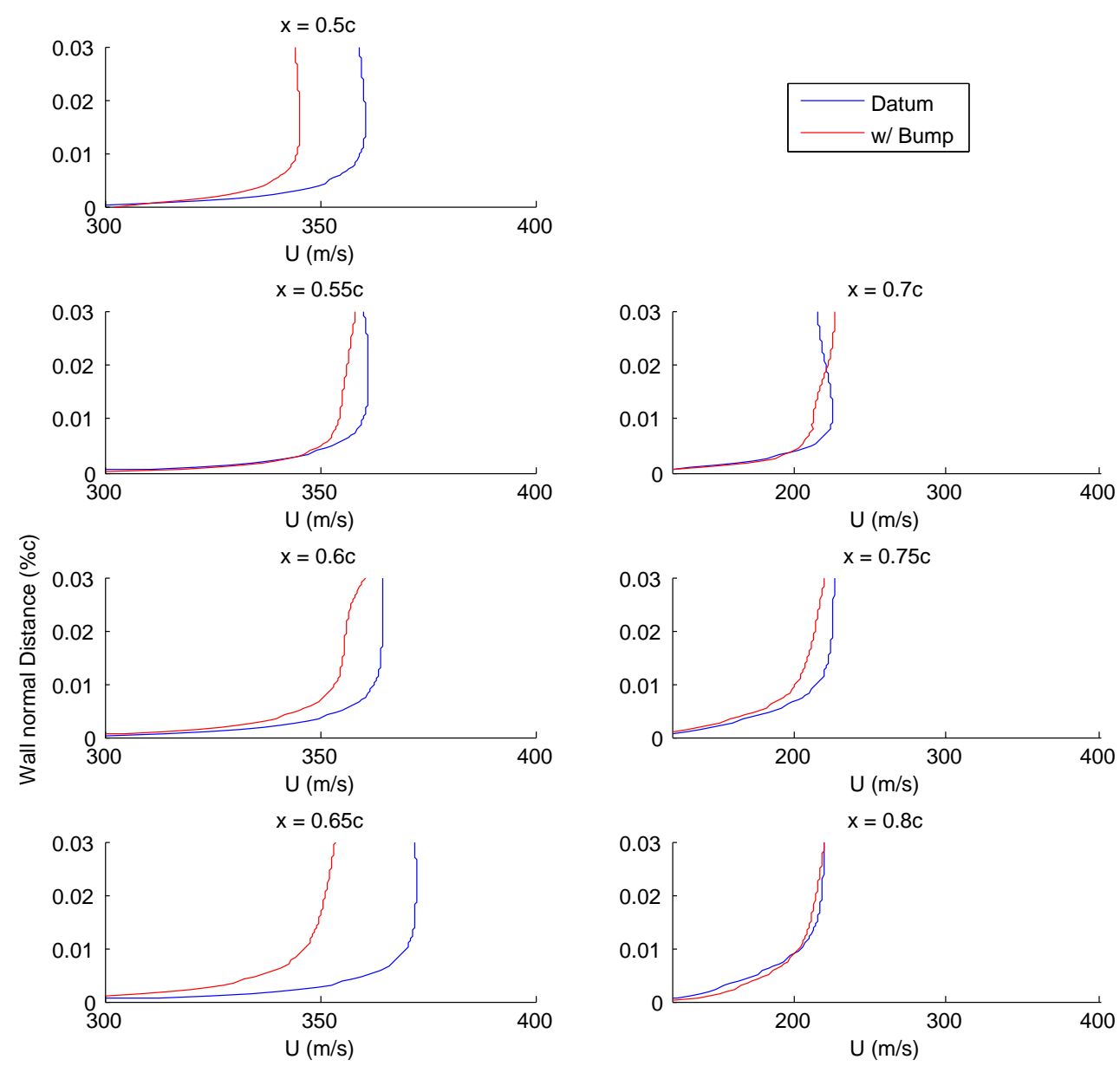

Figure 16. Boundary layer profiles surrounding SCB. The first column represents the pre-shock profiles and the second downstream of the shock. The locations are shown in figure 15 . 
of the square rod that can be seen at $0.56 l_{b}$, the shape of the corners will have to be analysed in order to give the most accurate displacements at various locations.

The adaptive SCB is fitted to the lower wall of the working section and consisted of a thin plate of high-strength AL-7075-T6 ( $\mathrm{t}=0.6 \mathrm{~mm}, \mathrm{E}=72 \mathrm{GPa}, \nu=0.33)$, which is to be deformed by a series of small actuators positioned to replicate the geometries produced here. The captive linear actuators are capable of producing $80 \mathrm{~N}$ throughout the stroke of $13 \mathrm{~mm}$. These can be seen in Figure 17 and will deform the 0.6 mm sheet of aluminium to create the shape of the bump.

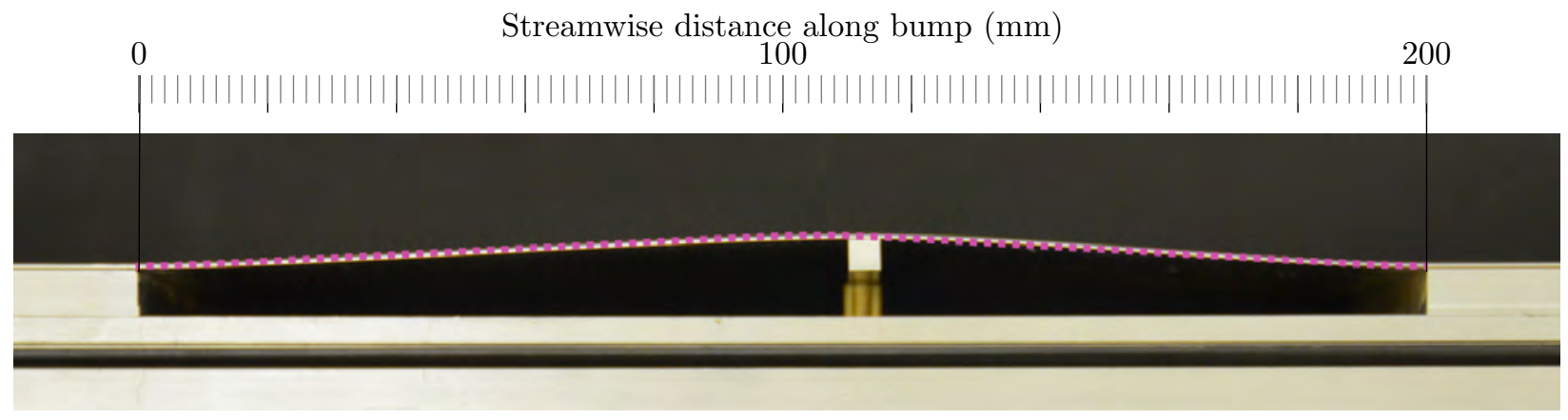

Figure 17. Experimental model of single crest bump in Table 3 . The finite element coordinates have been overlaid to compare the solutions.

Potential issues regarding the dissimilarity between the airfoil setup have been identified, in particular the pressure difference between the low pressure supersonic air in the tunnel and the stationary ambient air outside. This has been overcome with a sealing plate which will allow a pocket of air to remain under the flexible surface which will then equalize upon tunnel startup. The thickness of the sheet was tested for the maximum pressure difference of 0.6 Bar and should not suffer permanent deformation under loading.

By creating these bleed valves for the air under the bump to equalize upon startup they have also created a pathway for the high pressure air downstream of the shock to travel upstream, how much this affects the flow has yet to be seen however some sealing may be required even to limit the flow rather than eliminate it completely. The passage of these higher pressures may also have an effect on bump geometry and alter the shape with dependence upon the location of the rear leg of the shock and the corresponding pressure rise. This could then lead towards a passive flow control device whereby the thin sheet deforms creating a new family of SCB dependent entirely upon the shock location.

\section{Conclusions and Continuing Work}

The aero-structural optimization carried out has shown significant benefits when compared to the clean airfoil with a marginal $5 \%$ decrease in performance when compared to the optimal unconstrained bump. These constrained bumps, due to the presence of many local minima, appear to be much more versatile for a wider range of shock positions. This work suggests that constrained SCB are able to achieve $95 \%$ of the unconstrained optimal performance across a wider envelope of shock locations due to flight conditions. The adaptivity ensures that this positive influence is transferred beyond this range of small shock displacements and can be applied to appreciable movements in shock position due to significant changes in flight conditions.

- Structural constraints do limit the height of achievable bumps but have a wider range of favorable operating conditions even without adaptivity.

- Incorporation of adaptivity results in $95 \%$ of unconstrained optimal total pressure recovery over a larger flight envelope.

- Total pressure recovery has been successfully used as a performance metric and will provide a valuable comparison with future experimental data.

- Differences have been found between 2D/3D design protocols, single crest bumps may be just as efficient as table top designs without the added complexity. 
- Holding the shock position downstream of the crest increases triple point height and potentially increases total pressure recovery.

- A wind tunnel model has been developed for the experimental analysis of adaptive SCB with the computed geometry being very well replicated.

\section{References}

${ }^{1}$ Ashill, P., Fulker, J. L., and Shires, A., "A Novel Technique for Controllling Shock Strength of Laminar-Flow Aerofoil Sections," First European Forum on Laminar Flow Technology, 1992, pp. 175-183.

${ }^{2}$ Wadehn, W., Sommerer, A., Lutz, T., Fokin, D., Pritschow, G., and S.Wagner, "Structural Concepts and Aerodynamic Design of Shock Control Bumps," ICAS 2002 CONGRESS, 2002.

${ }^{3}$ Bein, T., Hanselka, H., and Breitbach, E., "An adaptive spoiler to control the transonic shock," Smart Materials and Structures, Vol. 9, No. 2, April 2000, pp. 141-148.

${ }^{4}$ Dargel, G., "Assessment of the Capability of Drag Reduction of the Shock Control Device Shock Bump on Airfoil Flows and Application Aspects on Wings," IUTAM Symposium on Mechanics of Passive and Active Flow Control, 1999, pp. 57-62.

${ }^{5}$ Bohning, R. and Stern, D., "Shock/Boundary Layer Control by Various Concepts," IUTAM Symposium on Mechanics of Passive and Active Flow Control, 1999, pp. 23-28.

${ }^{6}$ Bruce, P. J. K. and Babinsky, H., "An experimental study into the flow physics of three-dimensional shock control bumps," Journal of Aircraft, Vol. 49, No. 5, 2012, pp. 1222-1233.

${ }^{7}$ Colliss, S. P., Babinsky, H., Nübler, K., and Lutz, T., "Vortical structures on three-dimensional shock control bumps," 51st AIAA Aerospace Sciences Meeting including the New Horizons Forum and Aerospace Exposition, No. January, AIAA, Grapevine (Dallas/Ft. Worth Region), Texas, 2013, pp. 1-15.

${ }^{8}$ Klemens, N., Lutz, T., Krämer, E., Colliss, S. P., and Babinsky, H., "Shock Control Bump Robustness Enhancement," 50th AIAA Aerospace Sciences Meeting including the New Horizons Forum and Aerospace Exposition 09 - 12 January 2012, Nashville, Tennessee, No. January, 2012.

${ }^{9}$ Ogawa, H., Experimental and Analytical Investigation of Transonic Shock-wave / Boundary-layer Interaction Control with Three-dimensional Bumps, Ph.D. thesis, Clare Hall College, Cambridge University, 2006.

${ }^{10}$ Rosemann, E. S. and H., "Active Flow Control Applied to Military and Civil Aircraft," RTO AVT Symposium on Active Control Technology for Enhanced Performance Operational Capabilities of Military Aircraft, Land Vehicles and Sea Vehicles, held in Braunschweig, Germany, 8-11 May 2000, No. May, 2000, pp. 8-11.

${ }^{11}$ Rhodes, O. and Santer, M., "Aeroelastic Optimization of a Morphing 2D Shock Control Bump," 53rd AIAA/ASME/ASCE/AHS/ASC Structures, Structural Dynamics and Materials Conference 20th AI 23 - 26 April 2012, Honolulu, Hawaii AIAA 2012-1440, 2012, pp. 1-17.

12 "Abaqus CAE v6.10 DassaultSystèmes," 2007.

13 "OpenFoam ltd." 2013.

14 "MATLAB and Statistics Toolbox Release 2012b Mathworks inc." .

${ }^{15}$ Eastwood, J. P. and Jarrett, J. P., "Toward Designing with Three-Dimensional Bumps for Lift/Drag Improvement and Buffet Alleviation," AIAA Journal, Vol. 50, No. 12, Dec. 2012, pp. 2882-2898.

${ }^{16}$ Stanewsky, E., Délery, J., Fulker, J. L., and de Matteis, P., Drag Reduciton by Shock and Boundary Layer Control: Results of the Project EUROSHOCK II, Springer, 2002.

${ }^{17}$ Sommerer, A., Lutz, T., and Wagner, S., "Numerical Optimization of Adaptive Transonic Airfoils with Variable Camber," Proceedings 22nd ICAS Congress, Harrogate, UK, 2000.

${ }^{18}$ Sommerer, A., Th., L., and Wagner, S., "Design of Adaptive transonic Airfoils by Means of Numerical Optimization," Proceedings ECCOMAS 2000: European Congress on Computational Methods in Applied Sciences and Engineering, 2000, p. 379.

${ }^{19}$ Thompson, J. F., Warsi, Z. U. A., and Wayne Mastin, C., Numerical Grid Generation - Foundations and Applications, North-Holland, 1985.

${ }^{20}$ Cook, P., McDonald, M., and Firmin, M., "Aerofoil RAE 2822 - Pressure Distributions, and Boundary Layer and Wake Measurements," Experimental Data Base for Computer Program Assessment, AGARD Report AR 138., 1979.

${ }^{21}$ Wong, W., Qin, N., Sellars, N., Holden, H., and Babinsky, H., "A combined experimental and numerical study of flow structures over three-dimensional shock control bumps," Aerospace Science and Technology, Vol. 12, No. 6, Sept. 2008, pp. $436-447$.

${ }^{22}$ Qin, N., Wong, W. S., and Le Moigne, A., "Three-dimensional contour bumps for transonic wing drag reduction," Proceedings of the Institution of Mechanical Engineers, Part G: Journal of Aerospace Engineering, Vol. 222, No. 5, Aug. 2008, pp. 619-629.

${ }^{23}$ Colliss, S. P. and Babinsky, H., "An experimental investigation of $\mathrm{t}$ hree-dimensional shock control bumps applied to transonic airfoils," 50th AIAA Aerospace Sciences Meeting including the New Horizons Forum and Aerospace Exposition 09 12 January 2012, Nashville, Tennessee, No. January, 2012, pp. 1-15.

${ }^{24}$ Birkemeyer, J., Rosemann, H., and Stanewsky, E., "Shock control on a swept wing," Aerospace Science and Technology, Vol. 4, No. 3, April 2000, pp. 147-156.

${ }^{25}$ Pätzold, M., Krämer, E., Lutz, T., and Wagner, S., "Numerical Optimization of finite Shock Control Bumps," 44th Aerospace Sciences Meeting and Exhibit, 912 January 2006, Reno, Nevada, No. January, 2006.

${ }^{26}$ Lee, D., Bugeda, G., Periaux, J., and Onate, E., "Robust active shock control bump design optimisation using hybrid parallel MOGA," Computers \& Fluids, March 2012. 
${ }^{27}$ Donovan, J. F., Spina, E. F., and Smits, A. J., "The structure of a supersonic turbulent boundary layer subjected to concave surface curvature," Journal of Fluid Mechanics, Vol. 259, 1994, pp. 1-24. 